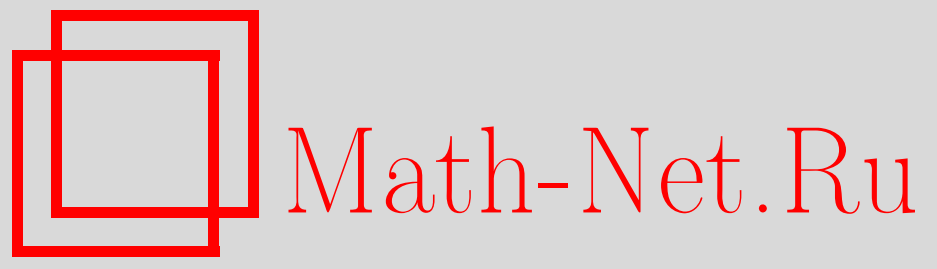

В. Г. Михайлов, Н. М. Меженная, Оценки для вероятности плотного вложения одной дискретной последовательности в другую, Дискрет. матем., 2005, том 17, выпуск 3, 19-27

DOI: https://doi.org/10.4213/dm113

Использование Общероссийского математического портала Math-Net.Ru подразумевает, что вы прочитали и согласны с пользовательским соглашением http: //www . mathnet.ru/rus/agreement

Параметры загрузки:

IP : 52.6 .47 .48

26 апреля 2023 г., 18:31:50

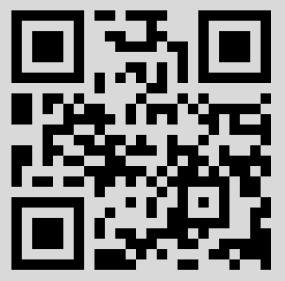


УдК 519.2

\title{
Оценки для вероятности плотного вложения одной дискретной последовательности в другую
}

\author{
( 2005 г. В. Г. Михайлов, Н. М. Меженная
}

\begin{abstract}
Получены верхняя и нижняя оценки для вероятности того, что заданная последовательность $X$ конечной длины из знаков конечного алфавита встретится в случайной равновероятной последовательности $Y$ знаков того же алфавита как подпоследовательность, члены которой разделены в $Y$ не более, чем одним знаком. Указаны последовательности $X$, на которых достигаются эти оценки.

Работа выполнена при поддержке Российского фонда фундаментальных исследований, проекты 02-01-00266 и 05.01.00035, и программы Президента Российской Федерации государственной поддержки ведущих научных школ, проект НШ-1758.2003.1.
\end{abstract}

\section{1. Введение}

Пусть $X_{n}=\left\{x_{i}\right\}_{i=1}^{n}$ и $Y_{m}=\left\{y_{i}\right\}_{i=1}^{m}-$ последовательности знаков алфавита $A_{N}=\{0,1, \ldots, N-1\}$. Согласно [1] последовательность $X_{n}$ плотно вкладывается в начало последовательности $Y_{m}$, если $n \leqslant m$ и найдутся такие натуральные числа

$$
1=j_{1}<j_{2}<\ldots<j_{n} \leqslant m, \quad j_{k+1}-j_{k} \in\{1,2\}, \quad k=1, \ldots, n-1,
$$

что $x_{k}=y_{j_{k}}, k=1, \ldots, n$ (в этом случае будем говорить, что $k$-й знак последовательности $X_{n}$ вставлен. в $Y_{m}$ на $j_{k}$-м месте).

В работе [1] была получена оценка сверху для вероятности $P_{m}\left(X_{n}\right)$ плотного вложения последовательности $X_{n}$ в последовательность $Y_{m}=\left\{y_{i}\right\}_{i=1}^{m}$ независимых случайных величин, распределенных равномерно на множестве $A_{2}$. В настоящей заметке получено обобщение этого результата на случай конечного алфавита $A_{N}$ с произвольным числом букв, показано, что эта верхняя оценка неулучшаема. Построена также неулучшаемая оценка снизу для вероятности $P_{m}\left(X_{n}\right)$. Основной результат работы следующий.

Теорема 1. Пусть знаки последовательности $Y_{m}=\left\{y_{i}\right\}_{i=1}^{m}$ распределены на множестве $A_{N}$ независимо и равномерно. Тогда

$$
\frac{1}{N^{n}} \sum_{k=0}^{m-n}\left(\begin{array}{c}
n-1 \\
k
\end{array}\right)\left(1-\frac{1}{N}\right)^{k} \leqslant P_{m}\left(X_{n}\right) \leqslant P_{2 n}\left(X_{n}\right),
$$


если $n \leqslant m<2 n, u$

$$
\begin{aligned}
\frac{1}{N^{2 n-1}}(2 N-1)^{n-1} & \leqslant P_{m}\left(X_{n}\right)=P_{2 n}\left(X_{n}\right) \\
& \leqslant \frac{1}{2 N^{2 n}}\left(\left(N-\sqrt{N^{2}-N}\right)^{n}+\left(N+\sqrt{N^{2}-N}\right)^{n}\right),
\end{aligned}
$$

если $2 n \leqslant m$.

Верхние оценки в этой теореме при $N=2$ совпадают с оценками работы [1]. Эти оценки при $m=2 n$ достигаются на последовательностях $X_{n}$, в которых нет совпадений соседних букв. Нижние оценки достигаются на последовательностях $X_{n}$, составленных из $n$ одинаковых букв.

\section{2. Доказательство верхних оценок}

Для доказательства теоремы 1 нам понадобятся дополнительные определения. Будем говорить, что последовательность $X_{n}=\left\{x_{i}\right\}_{i=1}^{n}$ может быть плотно вложена во всю последовательность $Y_{m}=\left\{y_{i}\right\}_{i=1}^{m}$, если помимо (1) выполняется соотношение $m-j_{n} \in\{1,2\}$ (в этом случае будем использовать обозначение $X_{n} \rightarrow Y_{m}$ ). При этом, естественно, $x_{n}=y_{m-1}$ или $x_{n}=y_{m}$. Если при плотном вложении $X_{n}$ в $Y_{m}$ знак $x_{n}$ вкладывается на $m$-е место последовательности $Y_{m}$, то будем говорить, что последовательность $X_{n}$ может быть плотно вложена в последовательность $Y_{m}$ с двумя прикрепленными концами (в этом случае используем обозначение $X_{n} \Rightarrow Y_{m}$ ).

Сначала рассмотрим случай $m \geqslant 2 n$. В этом случае только первые $2 n-1$ символов последовательности $\left\{y_{i}\right\}_{i=1}^{m}$ должны учитываться при вложении, а остальные могут быть произвольными. Значит,

$$
P_{m}\left(X_{n}\right)=\ldots=P_{2 n}\left(X_{n}\right)=P_{2 n-1}\left(X_{n}\right) .
$$

При построении верхней оценки удобно взять $m=2 n$.

Пусть

$$
B_{n, k, 0}\left(X_{n}\right)=\left|\left\{Y_{n+k}: X_{n} \Rightarrow Y_{n+k}\right\}\right|
$$

- число таких последовательностей $Y_{n+k}$ длины $n+k$, что $X_{n}$ может быть плотно вложена в $Y_{n+k}$ с двумя прикрепленными концами, а

$$
B_{n, k, 1}\left(X_{n}\right)=\left|\left\{Y_{n+k}: X_{n} \Rightarrow Y_{n+k-1}, X_{n} \nRightarrow Y_{n+k}\right\}\right|
$$

- число таких последовательностей $Y_{n+k}$ длины $n+k$, что $X_{n}$ может быть плотно вложена в $Y_{n+k-1}$ с двумя прикрепленными концами, но не может быть вложена с двумя прикрепленными концами в $Y_{n+k}$. При этом считаем, что $B_{n, k, i}\left(X_{n}\right)=0, i=0,1$, если $k<0$ или $k>n$.

При небольших значениях $n$ величина $B_{n, k, i}\left(X_{n}\right)$ легко вычисляется исходя из определения. Например, при $n=1$

$$
\begin{aligned}
& B_{1,0,0}\left(X_{1}\right)=\left|\left\{Y_{1}: X_{1} \Rightarrow Y_{1}\right\}\right|=\left|\left\{Y_{1}: X_{1}=Y_{1}\right\}\right|=1, \\
& B_{1,0,1}\left(X_{1}\right)=\left|\left\{Y_{0}: X_{1} \Rightarrow Y_{0}\right\}\right|=0, \\
& B_{1,1,0}\left(X_{1}\right)=\left|\left\{Y_{2}: X_{1} \Rightarrow Y_{2}\right\}\right|=0, \\
& B_{1,1,1}\left(X_{1}\right)=\left|\left\{Y_{2}: X_{1} \Rightarrow Y_{1}, X_{1} \nRightarrow Y_{2}\right\}\right|=\left|\left\{x_{1}=y_{1}, y_{2} \in A_{N}\right\}\right|=N .
\end{aligned}
$$


Теорема 2. Для любой последовательности $X_{n}$ длины $n$ из знаков алфавита $A_{N}$, для любого $k=0,1, \ldots, n$ выполнены соотночения

$$
\begin{aligned}
& B_{n, k, 0}\left(X_{n}\right)=B_{n-1, k, 0}\left(X_{n-1}\right)+B_{n-1, k, 1}\left(X_{n-1}\right), \\
& B_{n, k, 1}\left(X_{n}\right) \leqslant(N-1) B_{n, k-1,0}\left(X_{n}\right)+B_{n-1, k-1,1}\left(X_{n-1}\right)
\end{aligned}
$$

с начальными условиями

$$
B_{1,0,0}\left(X_{1}\right)=1, \quad B_{1,0,1}\left(X_{1}\right)=0, \quad B_{1,1,0}\left(X_{1}\right)=0, \quad B_{1,1,1}\left(X_{1}\right)=N .
$$

При этом (5) выполняется как равенство в случае $x_{n-1} \neq x_{n}$.

Теорема 2 будет доказана в разделе 4, а сейчас воспользуемся ею для вывода второго неравенства в (3).

Рассмотрим систему рекуррентных соотношений

$$
\begin{aligned}
& C_{n, k, 0}=C_{n-1, k, 0}+C_{n-1, k, 1}, \\
& C_{n, k, 1}=(N-1) C_{n, k-1,0}+C_{n-1, k-1,1}
\end{aligned}
$$

с начальными условиями

$$
C_{1,0,0}=1, \quad C_{1,0,1}=0, \quad C_{1,1,0}=0, \quad C_{1,1,1}=N .
$$

Она совпадает с системой (4), (5) в случае, когда неравенство (5) обращается в равенство. Из теоремы 2 следует, что

$$
C_{n, k, i} \geqslant B_{n, k, i}\left(X_{n}\right),
$$

и если в последовательности $X_{n}$ нет одинаковых подряд идущих знаков, то для таких последовательностей $C_{n, k, i}=B_{n, k, i}\left(X_{n}\right)$ при всех $n, k=0, \ldots, n, i=0,1$, так как начальные условия (6) не зависят от $X_{1}$.

Найдем $C_{n, k, i}$ при $n=2$, используя соотношения (7), (8) и начальные условия (9):

$$
\begin{aligned}
& C_{2,0,0}=C_{1,0}=1, \\
& C_{2,0,1}=0, \\
& C_{2,1,0}=C_{1,1,0}+C_{1,1,1}=N, \\
& C_{2,1,1}=(N-1) C_{2,0,0}+C_{1,0,1}=N-1, \\
& C_{2,2,0}=0, \\
& C_{2,2,1}=(N-1) C_{2,1,0}+C_{1,1,1}=N^{2} .
\end{aligned}
$$

Положим

$$
C_{n, k}=C_{n, k, 0}+C_{n, k, 1}
$$

Заметим, что величина $C_{n, k}$ определяет число таких последовательностей $Y_{n+k}$ длины $n+k$, что последовательность $X_{n}$ может быть плотно вложена во всю последовательность $Y_{n+k}$. Для величин $C_{n, k}$ при $n=1,2$ с учетом формулы (11) получим следующие значения:

$$
C_{1,0}=1, \quad C_{1,1}=N, \quad C_{2,0}=1, \quad C_{2,1}=2 N-1, \quad C_{2,2}=N^{2} .
$$


Лемма 1. Для любых $n \geqslant 3, k=0,1, \ldots, n$, выполнено соотночение

$$
C_{n, k}=C_{n-1, k}+N C_{n-1, k-1}-C_{n-2, k-1}
$$

с начальными условиями

$$
C_{1,0}=1, \quad C_{1,1}=N, \quad C_{2,0}=1, \quad C_{2,1}=2 N-1, \quad C_{2,2}=N^{2} .
$$

Доказательства этой и других лемм приведены в разделе 4.

Продолжим доказательство неравенства (3). Оценим число $D_{m}\left(X_{n}\right)$ детерминированных последовательностей $Y_{m}$, в начала которых может быть плотно вложена последовательность $X_{n}$. Назовем длиной вложения номер того элемента последовательности $Y_{m}$, которому (при вложении $X_{n}$ в $Y_{m}$ ) сопоставляется последний элемент последовательности $X_{n}$ (знак $x_{n}$ ). Для каждой пары последовательностей $X_{n}$ и $Y_{m}$ имеется хотя бы один вариант плотного вложения с максимальной длиной вложения.

Пусть $D_{m, k}\left(X_{n}\right)$ - число последовательностей $Y_{m}$, для которых максимальная длина плотного вложения $X_{n}$ в $Y_{m}$ равна $n+k$. Тогда

$$
D_{m}\left(X_{n}\right)=\sum_{k=0}^{m-n} D_{m, k}\left(X_{n}\right),
$$

причем

$$
D_{m, k}\left(X_{n}\right) \leqslant B_{n, k, 1}\left(X_{n}\right) N^{m-n-k} .
$$

Следовательно,

$$
D_{m}\left(X_{n}\right) \leqslant \sum_{k=0}^{n} N^{n-k} B_{n, k, 1}\left(X_{n}\right) .
$$

Воспользуемся теперь тем, что, как уже отмечалось (см. (10)), имеет место оценка $B_{n, k, 1}\left(X_{n}\right) \leqslant C_{n, k, 1}$. Приходим к неравенству

$$
D_{m}\left(X_{n}\right) \leqslant C_{n},
$$

где

$$
C_{n}=\sum_{k=0}^{m-n} C_{n, k, 1} N^{m-n-k}
$$

Поэтому

$$
P_{2 n}\left(X_{n}\right)=N^{-2 n} D_{2 n}\left(X_{n}\right) \leqslant N^{-2 n} C_{n} .
$$

Найдем величины $C_{1}$ и $C_{2}$, используя начальные условия (6) и определение (16):

$$
\begin{aligned}
& C_{1}=\sum_{k=0}^{1} N^{1-k} C_{1, k, 1}=N, \\
& C_{2}=\sum_{k=0}^{2} N^{2-k} C_{2, k, 1}=N^{2}+N .
\end{aligned}
$$

Далее воспользуемся следующим утверждением. 
Лемма 2. Для любого $n \geqslant 1$ величина $C_{n}$ выражается формулой

$$
C_{n}=\frac{1}{2}\left(N-\sqrt{N^{2}-N}\right)^{n}+\frac{1}{2}\left(N+\sqrt{N^{2}-N}\right)^{n} .
$$

Теперь верхняя оценка в (3) следует из (17) и (18).

Лемма 3. Пусть последовательность $X_{n}$ удовлетворяет условию

$$
x_{i} \neq x_{i+1}, \quad i=1,2, \ldots, n-1 .
$$

Тогда

$$
D_{m, k}\left(X_{n}\right)=C_{n, k, 1} N^{m-n-k} .
$$

Из равенства (20) и соотношений (14) и (16) следует, что для последовательности $X_{n}$, удовлетворяющей условию (19), выполнено равенство $D_{m}\left(X_{n}\right)=C_{n}$. Поэтому верхняя оценка в (3) для таких последовательностей превращается в равенство.

\section{3. Доказательство нижних оценок}

Выведем нижнюю оценку для вероятности $P_{m}\left(X_{n}\right)$ в предположении, что $m \geqslant 2 n$. Наряду с основным (описанным ранее) способом вложения последовательности $X_{n}$ в последовательность $Y_{m}$, рассмотрим также вспомогательный способ. При вспомогательном способе делаем следующее. Если $x_{1}=y_{1}$, то вставляем знак $x_{1}$ на первое место последовательности $Y_{m}$, в противном случае считаем, что последовательность $X_{n}$ не может быть вставлена в $Y_{m}$. Далее рассуждаем рекурсивно. Пусть знак $x_{i-1}$ вставлен в $Y_{m}$ на $(j-1)$-м месте. Если $x_{i}=y_{j}$, то вставляем знак $x_{i}$ на $j$-е место последовательности $Y_{m}$. Если $x_{i} \neq y_{j}, x_{i}=y_{j+1}$, то вставляем $x_{i}$ на $(j+1)$-е место. Если же $x_{i} \neq y_{j}, x_{i} \neq y_{j+1}$, то будем считать, что последовательность $X_{n}$ не может быть вставлена вспомогательным способом в $Y_{m}$.

Ясно, что если последовательность $X_{n}$ может быть вставлена в начало последовательности $Y_{m}$ вспомогательным способом, то она может быть вставлена в него и основным способом. Поэтому вероятность вложения последовательности $X_{n}$ в случайную последовательность $Y_{m}$ вспомогательным способом меньше или равна вероятности этого же вложения основным способом.

Лемма 4. Если последовательность $X_{n}$ состоит из $n$ одинаковых знаков, то вероятности вложения $X_{n}$ в случайную равновероятную последовательность $Y_{m}$ основньм $u$ вспомогательным способами совпадают.

Поэтому нижняя оценка вероятности $P_{m}\left(X_{n}\right)$ достигается для таких последовательностей $X_{n}$.

Вычислим вероятность вложения $X_{n}$ в $Y_{m}$ вспомогательным способом. Обозначим через $D_{m}^{\prime}\left(X_{n}\right)$ число детерминированных последовательностей $Y_{m}=\left\{y_{j}\right\}_{j=1}^{m}$, в которые данная последовательность $X_{n}=\left\{x_{j}\right\}_{j=1}^{n}$ может быть вставлена вспомогательным способом.

Лемма 5. Для любых $n \geqslant 1$ и $m \geqslant n$ выполнено равенство

$$
D_{m}^{\prime}\left(X_{n}\right)=\sum_{k=0}^{m-n}\left(\begin{array}{c}
n-1 \\
k
\end{array}\right)(N-1)^{k} N^{m-n-k} .
$$


Следствие 1. При $m \geqslant 2 n-1$ выполнено равенство

$$
D_{m}^{\prime}\left(X_{n}\right)=(2 N-1)^{n-1} N^{m-2 n+1} \text {. }
$$

Следствие 2. Значение $D_{m}^{\prime}\left(X_{n}\right)$ не зависит от $X_{n}$.

Нам остается доказать первое из неравенств (2), так как второе неравенство очевидно. Таким образом, мы переходим к рассмотрению случая $n \leqslant m<2 n$.

Итак, мы можем в качестве нижней оценки для вероятности $P_{m}\left(X_{n}\right)$ взять выражение $N^{-m} D_{m}^{\prime}\left(X_{n}\right)$. Согласно лемме 5 и следствиям из нее такая нижняя оценка вероятности $P_{m}\left(X_{n}\right)$ не зависит от $X_{n}$, достигается на последовательностях из одинаковых знаков и имеет вид

$$
\begin{aligned}
P_{m}\left(X_{n}\right) \geqslant \frac{1}{N^{m}} D_{m}^{\prime}\left(X_{n}\right) & =\frac{1}{N^{m}} \sum_{k=0}^{m-n}\left(\begin{array}{c}
n-1 \\
k
\end{array}\right)(N-1)^{k} N^{m-n-k} \\
& =\frac{1}{N^{n}} \sum_{k=0}^{m-n}\left(\begin{array}{c}
n-1 \\
k
\end{array}\right)\left(1-\frac{1}{N}\right)^{k}
\end{aligned}
$$

Таким образом, теорема 1 доказана.

\section{4. Доказательства вспомогательных утверждений}

Доказательство теоремы 2. Используем такую же схему рассуждений, как в работе [1]. Заметим, что при $0 \leqslant k \leqslant n$ имеет место равенство

$$
\begin{aligned}
\left\{X_{n}\right. & \left.\Rightarrow Y_{n+k}\right\}=\left\{y_{n+k}=x_{n}, X_{n-1} \rightarrow Y_{n+k-1}\right\} \\
& =\left\{y_{n+k}=x_{n}, X_{n-1} \Rightarrow Y_{n+k-1}\right\} \cup\left\{y_{n+k}=x_{n}, X_{n-1} \Rightarrow Y_{n+k-2}, X_{n} \nRightarrow Y_{n+k-1}\right\} .
\end{aligned}
$$

В силу несовместности последних событий мы получим (4).

Докажем формулу (5). Разложим событие $\left\{X_{n} \Rightarrow Y_{n+k-1}, X_{n} \nRightarrow Y_{n+k}\right\}$ в сумму двух несовместных событий:

$$
\begin{aligned}
\left\{X_{n} \Rightarrow Y_{n+k-1}, X_{n} \nRightarrow Y_{n+k}\right\}=\left\{y_{n+k} \neq x_{n}, X_{n} \Rightarrow Y_{n+k-1}, X_{n} \nRightarrow Y_{n+k}\right\} \\
\cup\left\{y_{n+k}=x_{n}, X_{n} \Rightarrow Y_{n+k-1}, X_{n} \nRightarrow Y_{n+k}\right\} .
\end{aligned}
$$

Ясно, что

$$
\left\{y_{n+k} \neq x_{n}, X_{n} \Rightarrow Y_{n+k-1}, X_{n} \nRightarrow Y_{n+k}\right\}=\left\{y_{n+k} \neq x_{n}, X_{n} \Rightarrow Y_{n+k-1}\right\} .
$$

Неравенство (5) будет доказано, если мы убедимся, что

$$
\begin{aligned}
\left\{y_{n+k}=x_{n}, X_{n} \Rightarrow\right. & \left.Y_{n+k-1}, X_{n} \nRightarrow Y_{n+k}\right\} \\
& \subseteq\left\{X_{n-1} \Rightarrow Y_{n+k-3}, X_{n-1} \nRightarrow Y_{n+k-2}\right\} \cap\left\{y_{n+k-1}=y_{n+k}=x_{n}\right\} .
\end{aligned}
$$


Для этого перепишем событие в левой части последнего включения следующим образом:

$$
\begin{aligned}
& \left\{y_{n+k}=x_{n}, X_{n} \Rightarrow Y_{n+k-1}, X_{n} \nRightarrow Y_{n+k}\right\} \\
& =\left\{X_{n} \Rightarrow Y_{n+k-1}, X_{n-1} \nRightarrow Y_{n+k-1}, X_{n-1} \nRightarrow Y_{n+k-2}\right\} \cap\left\{x_{n}=y_{n+k}\right\} \\
& =\left\{X_{n} \Rightarrow Y_{n+k-1}, X_{n-1} \nRightarrow Y_{n+k-1}, X_{n-1} \nRightarrow Y_{n+k-2}, X_{n-1} \Rightarrow Y_{n+k-3}\right\} \cap\left\{x_{n}=y_{n+k}\right\} \\
& =\left\{X_{n} \Rightarrow Y_{n+k-1}, X_{n-1} \nRightarrow Y_{n+k-2}, X_{n-1} \Rightarrow Y_{n+k-3}, x_{n}=y_{n+k}\right\} \\
& \quad \backslash\left\{X_{n} \Rightarrow Y_{n+k-1}, X_{n-1} \Rightarrow Y_{n+k-1}, x_{n}=y_{n+k}\right\} \\
& =\left\{X_{n-1} \nRightarrow Y_{n+k-2}, X_{n-1} \Rightarrow Y_{n+k-3}, x_{n}=y_{n+k}=y_{n+k-1}\right\} \\
& \quad \backslash\left\{X_{n} \Rightarrow Y_{n+k-1}, X_{n-1} \Rightarrow Y_{n+k-1}, x_{n}=y_{n+k}\right\} .
\end{aligned}
$$

При этом $\left\{X_{n} \Rightarrow Y_{n+k-1}, X_{n-1} \Rightarrow Y_{n+k-1}, x_{n}=y_{n+k}\right\}=\varnothing$, если $x_{n-1} \neq x_{n}$.

Из последнего равенства следует, что

$$
\begin{aligned}
\left\{y_{n+k}=x_{n}, X_{n} \Rightarrow Y_{n+k-1},\right. & \left.X_{n} \nRightarrow Y_{n+k}\right\} \\
& \subseteq\left\{X_{n-1} \nRightarrow Y_{n+k-2}, X_{n-1} \Rightarrow Y_{n+k-3}, x_{n}=y_{n+k}=y_{n+k-1}\right\} .
\end{aligned}
$$

В случае, когда $x_{n-1} \neq x_{n}$, это включение выполняется в форме равенства.

Таким образом, неравенство (5) доказано. Начальные условия были получены нами ранее. Доказательство теоремы 2 завершено.

Доказательство леммы 1. Из соотношения (7) вытекает, что

$$
C_{n, k, 0}=C_{n-1, k} .
$$

Поэтому с учетом уравнения (7) получаем, что

$$
C_{n, k, 1}=(N-1) C_{n-1, k-1}+C_{n-1, k-1,1}
$$

Из соотношения (11) находим, что $C_{n-1, k-1}=C_{n-1, k-1,0}+C_{n-1, k-1,1}$. Следовательно, с учетом равенства (22), $C_{n-1, k-1,1}=C_{n-1, k-1}-C_{n-2, k-1}$. Подставив этот результат в уравнение (23), получим, что

$$
C_{n, k, 1}=(N-1) C_{n-1, k-1}+C_{n-1, k-1}-C_{n-2, k-1}=N C_{n-1, k-1}-C_{n-2, k-1} .
$$

После этого из формулы (11) следует, что

$$
C_{n, k}=C_{n-1, k}+N C_{n-1, k-1}-C_{n-2, k-1} .
$$

Лемма 1 доказана.

Доказательство леммы 2. Пусть

$$
C_{n}^{\prime}=\sum_{k=0}^{n} N^{n-k} C_{n, k} .
$$

Воспользуемся выражением (12) для величины $C_{n, k}$, тогда

$$
\begin{aligned}
C_{n}^{\prime} & =\sum_{k=0}^{n} N^{n-k} C_{n, k}=\sum_{k=0}^{n} N^{n-k}\left(C_{n-1, k}+N C_{n-1, k-1}-C_{n-2, k-1}\right) \\
& =2 N C_{n-1}^{\prime}-N C_{n-2}^{\prime} .
\end{aligned}
$$


Из определения $C_{n}^{\prime}$ следует, что

$$
C_{n}^{\prime}=C_{n}+\sum_{k=0}^{n} N^{n-k} C_{n, k, 0} .
$$

Второе слагаемое с учетом уравнения (4) равно

$$
\sum_{k=0}^{n} N^{n-k} C_{n-1, k}=N C_{n-1}^{\prime} .
$$

Поэтому

$$
C_{n}=C_{n}^{\prime}-N C_{n-1}^{\prime}
$$

Ясно, что $C_{n}$ удовлетворяет тому же рекуррентному соотношению, что и $C_{n}^{\prime}$. Следовательно,

$$
C_{n}=2 N C_{n-1}-N C_{n-2} .
$$

Как известно (см. [2]), решение рекуррентного соотношения (24) следует искать в виде

$$
C_{n}=k_{1} q_{1}^{n}+k_{2} q_{2}^{n} \text {, }
$$

где $q_{1}, q_{2}-$ корни квадратного уравнения $q^{2}-2 N q+N=0$. Так как

$$
q_{1}=N-\sqrt{N^{2}-N}, \quad q_{2}=N+\sqrt{N^{2}-N},
$$

общее решение уравнения (24) имеет вид

$$
C_{n}=k_{1}\left(N-\sqrt{N^{2}-N}\right)^{n}+k_{2}\left(N+\sqrt{N^{2}-N}\right)^{n},
$$

где константы $k_{1}, k_{2}$ находятся из начальных условий и равны $k_{1}=k_{2}=1 / 2$.

Лемма 2 доказана.

Доказательство леммы 3. Заметим, что неравенство

$$
D_{m, k}\left(X_{n}\right)<B_{n, k, 1}\left(X_{n}\right) N^{m-n-k}
$$

возможно лишь в том случае, когда наряду с теми последовательностями $Y_{m}$, для которых максимальная длина плотного вложения $X_{n}$ в $Y_{m}$ равна $n+k$, имеется последовательность $Y_{m}$, в которую возможно вложение с длиной, не меньшей $n+k+2$. Покажем, что для последовательности $X_{n}$, удовлетворяющей условию (19), это невозможно.

Пусть существует два разных варианта плотного вложения такой последовательности $X_{n}$ в начало $Y_{m}$ и $n_{1}<n_{2}<\ldots<n_{r} \leqslant n$ - номера тех элементов $X_{n}$, которые вкладываются этими способами одинаково. В частности, $n_{1}=1$.

Заметим, что знаки $x_{2}, \ldots, x_{n_{2}-1}$ каждым их этих вариантов могут быть вложены лишь поочередно: на места $2,4, \ldots, 2 n_{1}-2$ и на места $3,5, \ldots, 2 n_{1}-3$ соответственно, а знак $x_{n_{2}}$ вставляется на место с номером $2 n_{2}-1$. То же самое выполняется для всех остальных элементов последовательности $X_{n}$. Поэтому длины вложения этими способами могут различаться не более, чем на единицу. Значит, неравенство (25) невозможно, и для таких последовательностей $X_{n}$

$$
D_{m, k}\left(X_{n}\right)=B_{n, k, 1}\left(X_{n}\right) N^{m-n-k} .
$$

Осталось воспользоваться теоремой 2 , согласно которой для рассматриваемых последовательностей $X_{n}$ выполнено равенство $B_{n, k, 1}\left(X_{n}\right)=C_{n, k, 1}$. Лемма 3 доказана. 
Доказательство леммы 4. Рассмотрим последовательность $X=(a, \ldots, a)$, которая может быть вставлена в начало последовательности $Y_{m}$ основным способом. В этом случае множество $\left\{1 \leqslant i \leqslant m: y_{i}=a\right\}$ состоит не менее, чем из $n$ элементов, причем первые $n$ из них удовлетворяют условию (1). Следовательно, последовательность $X_{n}$ может быть вставлена именно на эти первые места. Это означает, что последовательность $X_{n}$ вставляется в начало последовательности $Y_{m}$ вспомогательным способом.

Как мы уже отмечали, если последовательность $X_{n}$ может быть вставлена в начало последовательности $Y_{m}$ вспомогательным способом, то она может быть вставлена в него и основным способом. Таким образом, вероятности вставить последовательность $X_{n}$ указанного вида в начало последовательности $Y_{m}$ обоими способами совпадают. Лемма 4 доказана.

Доказательство леммы 5. Для любых $X_{n}$ и $Y_{m}$ существует не более одного варианта вставки последовательности $X_{n}$ в последовательность $Y_{m}$ вспомогательным способом. Это свойство позволяет найти число $D_{m}^{\prime}\left(X_{n}\right)$ следующим образом. Выберем число $k$, $0 \leqslant k \leqslant n-1$ и будем считать, что из $n$ знаков последовательности $X_{n}$ любые $n-k$ знаков вставлены вспомогательным способом сразу за предшествующим вставленным знаком последовательности $X_{n}$, а остальные $k$ знаков вставлены через пропуск знака. Число последовательностей $Y_{n+k}$ длины $n+k$, в которые данная последовательность $X_{n}$ может быть вставлена вспомогательным образом, равно $\left(\begin{array}{c}n-1 \\ k\end{array}\right)(N-1)^{k}$. Последние $m-n-k$ символов последовательности $Y_{m}$ могут быть выбраны произвольно, то есть $N^{m-n-k}$ способами. В итоге, суммируя по $k$ в диапазоне от 0 до $m-n$, получаем, что

$$
D_{m}^{\prime}\left(X_{n}\right)=\sum_{k=0}^{m-n}\left(\begin{array}{c}
n-1 \\
k
\end{array}\right)(N-1)^{k} N^{m-n-k}
$$

Лемма 5 доказана.

Авторы признательны А. М. Зубкову, обратившему их внимание на рассматриваемую задачу и на статью [1], а также А. А. Ахметшину за идею доказательства леммы 3.

\section{Список литературы}

1. Golic J. D., Constrained embedding probability for two binary strings. SIAM J. Discrete Math. (1996) 9, №3, 360-364.

2. Холл М., Комбинаторика. Мир, Москва, 1970.

Статья поступила 30.11.2004. 